\title{
OBJECTIVE ASSESSMENT OF IMAGE QUALITY IN CONVENTIONAL AND DIGITAL MAMMOGRAPHY TAKING INTO ACCOUNT DYNAMIC RANGE
}

\author{
Marc Pachoud ${ }^{1}$, Domenico Lepori ${ }^{2}$, Jean-François Valley ${ }^{1}$ and Francis R. Verdun ${ }^{1, *}$ \\ ${ }^{1}$ University Institute for Applied Radiophysics, Grand-Pré 1, CH-1007 Lausanne, Switzerland \\ ${ }^{2}$ Department of Diagnostic and Interventional Radiology, Lausanne University Hospital (CHUV), \\ CH-1011 Lausanne, Switzerland
}

\begin{abstract}
The goal of this work is to develop a method to objectively compare the performance of a digital and a screen-film mammography system in terms of image quality. The method takes into account the dynamic range of the image detector, the detection of high and low contrast structures, the visualisation of the images and the observer response. A test object, designed to represent a compressed breast, was constructed from various tissue equivalent materials ranging from purely adipose to purely glandular composition. Different areas within the test object permitted the evaluation of low and high contrast detection, spatial resolution and image noise. All the images (digital and conventional) were captured using a CCD camera to include the visualisation process in the image quality assessment. A mathematical model observer (nonprewhitening matched filter), that calculates the detectability of high and low contrast structures using spatial resolution, noise and contrast, was used to compare the two technologies. Our results show that for a given patient dose, the detection of high and low contrast structures is significantly better for the digital system than for the conventional screen-film system studied. The method of using a test object with a large tissue composition range combined with a camera to compare conventional and digital imaging modalities can be applied to other radiological imaging techniques. In particular it could be used to optimise the process of radiographic reading of soft copy images.
\end{abstract}

\section{INTRODUCTION}

The need for image quality assessments and optimisation in mammography is well established and is often based on the use of test objects ${ }^{(1,2)}$. One of the limitations of most of these test objects is that they are of uniform thickness and composition and do not address the issue of the variation of image quality according to tissue composition variations. To overcome this limitation, a test object has been recently proposed ${ }^{(3)}$. It contains various breast tissue equivalents allowing image quality assessments in a large dynamic range for high and low contrast structures. Moreover it has been shown that this new test object was adequate to assess image quality provided by conventional (i.e. using screen-film systems) as well as by digital mammography units. Image quality can be objectively assessed on digitised films or using the raw data when dealing with digital systems.

The goal of this work is to present a methodology allowing the assessment of image quality taking into account the display process of the images and the observer responses using a CCD camera to capture the signal, which impinges on the eyes of the reader of the images.

\section{MATERIALS AND METHODS}

The test object used in this study has been fully described elsewhere ${ }^{(3)}$ and was manufactured by

${ }^{*}$ Corresponding author: Francis.Verdun@chuv.ch
CIRS (Computerized Imaging Reference Systems, Norfolk, VA). It is made of breast tissue equivalent material and has a similar shape to a compressed breast (Figure 1). A $35 \mathrm{~mm}$ base layer of 50:50 glandular/adipose tissue equivalent is surrounded by a $5 \mathrm{~mm}$ shell of $100 \%$ adipose equivalent material to simulate the absorption of the skin (total thickness $45 \mathrm{~mm}$ ). Three areas within the test-object simulate the absorption of $100 \%$ glandular, 50:50 glandular/adipose, and 100\% adipose tissue. Low contrast target next to the $100 \%$ glandular and 50:50 glandular/adipose areas are produced by $5 \mathrm{~mm}$ thick slabs of 75:25 glandular/adipose tissue equivalent. A $5 \mathrm{~mm}$ slab of 25:75 glandular/adipose tissue gives a third low contrast target next to the $100 \%$ adipose area.

Films were made using a Bennett Contour 2000 unit (TREX medical, Danbury CT) with molybdenum anode and molybdenum filtration $(\mathrm{Mo} / \mathrm{Mo})$ and a high voltage of $28 \mathrm{kV}$. The film-screen combination was Kodak MinR 2190-MinR-L. Test object exposures were such that the average optical density at $60 \mathrm{~mm}$ from the chest wall in the 50:50 glandular/ adipose area was equal to $1.45 \pm 0.05$. Digital images were acquired on a Senographe 2000D unit (General Electric, USA) using the same exposure conditions as for the film (manual exposure, Mo/Mo combination). All the measurements were performed on raw images (non-equalised, non-filtered data).

The films were displayed onto a 'SmartLight' digital viewbox (SmartLight, Yokneam Industrial Park, Israel). Digital images were displayed using a 


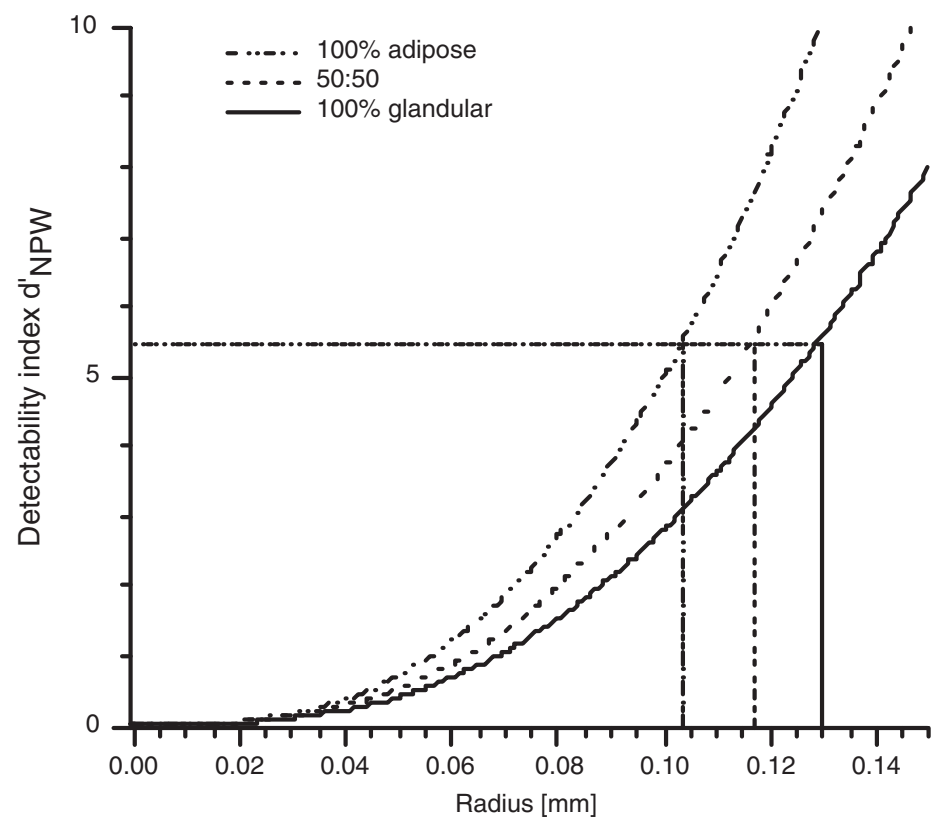

Figure 1. Variations in the detectability index, as a function of the diameter of a spherical high contrast target in the three breast tissue equivalent parts of the test object measured with the camera on a film exposed at $28 \mathrm{kV}$ with a Mo/Mo anode-filter combination.

cathode ray tube (CRT) display system in portrait mode (SMM-21140P model; Siemens AG Medical, Munich, Germany), having a maximal resolution of $1280 \times 1600$ pixels and connected to a DOME Md2/PCI graphic board (DOME imaging systems, inc, Waltham, MA). A PMI-1401 C camera, manufactured by ' $Q$ Imaging' ( $Q$ Imaging, Burnaby, BC, Canada), was used to capture all images involved in this study. It provides a digital output on 14 bits and contains a Kodak CCD (charged coupled device) made of $1317 \times 1035$ pixels (pixel size $6.8 \times 6.8 \mu \mathrm{m}^{2}$ ). The linearity of the output of the camera with the luminance detected has been verified for each situation (i.e. digital viewbox and CRT system) to assure a proper image contrast transfer. Moreover, the MTF of the camera has also been measured and the result has been used to deconvolve the final results. To qualify image quality by only one number for each image, the detectability index $\mathrm{d}_{\mathrm{NPW}}^{\prime}$ of the nonprewhitening matched filter model observer [corrected for the response of the eye (NPWE model)] ${ }^{(4,5)}$ was calculated. This model is based on the statistical decision theory that computes the probability distribution of the true-positive and the false-positive decisions obtained for a given decision task using a likelihood criterion. These probabilities are expressed as a function of a signal-to-noise ratio (SNR) which takes into account all the characteristics of the image system (i.e. the contrast, noise and MTF parameters captured by the camera). The SNR obtained when using the NPWE model observer represents the quantity $\mathrm{d}_{\mathrm{NPW}}^{\prime}$. To assure a detection with a reasonably high level of confidence, a fixed detectability index $\mathrm{d}_{\mathrm{NPW}}^{\prime}$ of 5.5 was chosen.

\section{RESULTS AND DISCUSSION}

The detectability index $\mathrm{d}_{\mathrm{NPW}}^{\prime}$ as a function of the diameter of a spherical high contrast target in the three breast tissue equivalent parts of the test object are presented in Figure 1. The measurements were performed with the camera on a film placed onto the viewbox. The results show that the detection of the sphere is easier in the fully adipose breast equivalent part of the test object than in the fully glandular breast equivalent part. Thus, a detectability index of 5.5 is already reached when the radius of the high contrast target is equal to $0.104 \mathrm{~mm}$ in the fully adipose breast equivalent part of the test object, while it requires a radius of $0.130 \mathrm{~mm}$ in the fully glandular breast equivalent part of the test object. This behaviour can be explained by the lower film contrast value in the glandular part than in the fully adipose part.

Similar calculations have been performed on the images displayed on the monitor, for the high and low contrast targets. The ratio of the radius obtained when dealing with the films with the 


\section{PACHOUD ET AL}

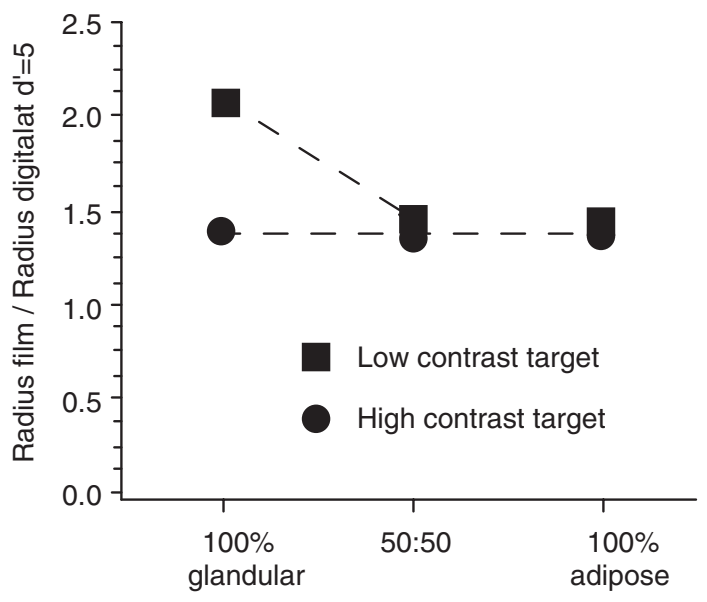

Figure 2. Ratio of the radius obtained with the film with the radius obtained when assessing the image quality of the image displayed on the monitor for a detectability index of 5.5 in the three tissue compositions of the test object.

one obtained when dealing with the soft copies has been obtained for the three tissue compositions of the test object. The results are presented in Figure 2.

No major difference appears when dealing with the high contrast target as a function of the tissue composition. The digital system allows the detection of a high contrast target $\sim 1.5$ smaller than the one that could be detected on the films. This nondependency on tissue composition can be explained by the high contrast level, which exists between the targets and the soft tissue elements. As regards the low contrast target it appears that the digital system is significantly better in the glandular part as in the two other tissues composition (i.e. 50\% glandular/ $50 \%$ adipose; $100 \%$ adipose).

As expected from the methodology, it appeared that the window widths and levels chosen to perform the measurements had only a minor impact (i.e. $\pm 10 \%)$ on the value of the detectability index.

\section{CONCLUSIONS}

We have developed a methodology that allows us to objectively assess image quality in the domain of the luminance which impinges on the eye of the observer. This methodology not only allows the comparison of the image quality of film with softcopy, but also the optimisation of the reading conditions of radiological images.

\section{ACKNOWLEDGEMENT}

The authors gratefully acknowledge the financial support of the Swiss Federal Office for Education and Science (grant no. 99.0739-Fifth European Research programme no. FIGMCT 2000 00036).

\section{REFERENCES}

1. van Voudenberg, S., Thijssen, M. and Young, K. European guidelines for quality assurance in mammography screening. Report EUR 14821, 3rd edition (Luxembourg: CEC) (1999).

2. Hendrick, R. E. and Berns, E. A. Optimizing mammographic technique. In: Proceedings of the RSNA Categorical Course in Breast Imaging, Chicago, IL, pp. 79-80 (1999).

3. Pachoud, M., Lepori, D., Valley, J.-F. and Verdun, F.R. A new test phanton with different breast tissue compositions for image quality assessment in conventional and digital mammography. Phys. Med. Biol. 49(23), 52675281 (2004).

4. Burgess, A. E., Jacobson, F. L. and Judy, P. F. Human observer detection experiments with mammograms and power-law noise. Med. Phys. 28(4), 419-437 (2001).

5. Bochud, F. O., Valley, J.-F., Verdun, F. R., Hessler, C. and Schnyder, P. Estimation of the noisy component of anatomical backgrounds. Med. Phys. 26(7), 1365-1370 (1999). 